\title{
Single or triple gradients?
}

\author{
Riddhiman Sarkar $^{\mathrm{a}}$, Detlef Moskau ${ }^{\mathrm{b}}$, Fabien Ferrage ${ }^{\mathrm{c}}$, Paul R. Vasos ${ }^{\mathrm{a}, *}$, Geoffrey Bodenhausen ${ }^{\mathrm{a}, \mathrm{c}}$ \\ a Institut des Sciences et Ingénierie Chimiques, Ecole Polytechnique Fédérale de Lausanne (EPFL), Batochime, Lausanne 1015, Vaud, Switzerland \\ ${ }^{\mathrm{b}}$ Bruker Biospin AG, NMR Division, Industriestrasse 26, Fällanden $\mathrm{CH}-8117$, Switzerland \\ ${ }^{\mathrm{C}}$ Département de Chimie, associé au CNRS, Ecole Normale Supérieure, 24 Rue Lhomond, 75231 Paris Cedex 05, France
}

\section{A R T I C L E I N F O}

Article history:

Received 12 March 2008

Revised 19 April 2008

Available online 24 April 2008

\section{Keywords:}

Pulsed field gradients

Diffusion

Coherence selection

Long-lived states

Singlet states

Triple gradients

Cryoprobe

\begin{abstract}
A B S T R A C T
Pulsed Field Gradients (PFGs) have become ubiquitous tools not only for Magnetic Resonance Imaging (MRI), but also for NMR experiments designed to study translational diffusion, for spatial encoding in ultra-fast spectroscopy, for the selection of desirable coherence transfer pathways, for the suppression of solvent signals, and for the elimination of zero-quantum coherences. Some of these experiments can only be carried out if three orthogonal gradients are available, while others can also be implemented using a single gradient, albeit at some expense of performance. This paper discusses some of the advantages of triple- with respect to single-gradient probes. By way of examples we discuss (i) the measurement of small diffusion coefficients making use of the long spin-lattice relaxation times of nuclei with low gyromagnetic ratios $\gamma$ such as nitrogen-15, and (ii) the elimination of zero-quantum coherences in Exchange or Nuclear Overhauser Spectroscopy (EXSY or NOESY) experiments, as well as in methods relying on long-lived (singlet) states to study very slow exchange or diffusion processes.
\end{abstract}

(c) 2008 Elsevier Inc. All rights reserved.

\section{Introduction}

Among the key ingredients that have made Magnetic Resonance Imaging (MRI) possible are carefully engineered coils designed to induce accurate Pulsed Field Gradients (PFGs). Three orthogonal gradients $G_{x}=\mathrm{d} B_{z} / \mathrm{d} x, G_{y}=\mathrm{d} B_{z} / \mathrm{dy}$, and $G_{z}=\mathrm{d} B_{z} / \mathrm{d} z$ can be generated by suitable Maxwell or Helmholtz coils designed to avoid generating non-linear (quadratic and higher) derivatives of the main $B_{z}$ field [1]. Such coils have evolved from shim-coils that were originally designed to improve the homogeneity of static fields for high-resolution spectroscopy, and from PFG coils that were designed to measure diffusion coefficients [2,3]. Ideally, the orthogonality of modern PFG coils precludes mutual interference, and socalled active shields, i.e., coils with similar geometry but larger dimensions and opposite polarity, make it possible to minimize the magnetic coupling with the environment, particularly with the innermost shield of the main magnet bore. Once optimized for MRI, sophisticated designs for PFG coils were soon 're-imported' into high-resolution spectroscopy. They have now become ubiquitous tools for the accurate measurement of translational diffusion coefficients, for spatial encoding in ultra-fast NMR, for elimination of unwanted zero-quantum coherences, for suppression of strong solvent signals [4], and for selection of coherence transfer pathways, either simply by dephasing or 'purging' unwanted coherences, or by selecting suitable coherence transfer echoes [5]

\footnotetext{
* Corresponding author. Fax: +41216939435.

E-mail address: paul.vasos@epfl.ch (P.R. Vasos).
}

to retain desirable pathways. In some instances, the use of gradients makes extensive phase cycles superfluous, e.g., in the case of heteronuclear correlations, a pair of gradients with strengths inversely proportional to the gyromagnetic ratios of the two involved nuclei ensures clean selection of the desired pathway. In homonuclear systems, the selection of coherences is in some cases impossible to perform using phase cycles alone. For instance, the distinction between longitudinal two-spin order and zero-quantum coherences requires pulsed field gradients.

Although some of these methods can only be applied if three orthogonal gradients are available, in other cases it has been possible to design a poor man's version using a single gradient. The limitation to a single gradient may lead to a sacrifice in performance, e.g., to imperfect selection of coherence transfer pathways. Poor suppression of water signals makes some experiments very difficult to implement on single-gradient probes, even when cumulative dephasing of the water magnetization is achieved by an appropriate choice of the signs of consecutive gradients [6]. Ultra-fast three- or four-dimensional NMR $[7,8]$ requires two or three orthogonal gradients. The simultaneous activation of two or three orthogonal gradients makes it possible to generate tilted gradients, in a manner that may allow one to control deleterious effects of demagnetizing dipolar fields [9-11]. On the other hand, triple gradients may entail a minor loss of sensitivity due to unavoidable technical compromises in the design of very crowded probes. Similar issues may arise for solid-state NMR with Magic Angle Spinning (MAS): while a single PFG with a tilted gradient $G_{z^{\prime}}=\mathrm{d} B_{\mathrm{z}} / \mathrm{d} z$ parallel to the spinning axis $z^{\prime}$ is reasonably straightforward to 
implement, perpendicular, rotor-synchronized, time-dependent gradients designed to appear static in the frame of the rotor are more challenging [12].

This paper discusses advantages of triple- over single-gradient probes. By way of example, we shall discuss two applications of broad interest:

(i) The encoding and decoding of the phases of coherences by gradients

This is relevant both for spin-echo diffusion experiments and for heteronuclear correlation experiments. We shall discuss the measurement of small diffusion coefficients via longitudinal magnetization of low- $\gamma$ nuclei [13] that have long spin-lattice relaxation times $T_{1}$, such as nitrogen-15;

(ii) The elimination of zero-quantum coherences while retaining longitudinal magnetization terms

Both EXSY and NOESY experiments [14-16] are discussed, as well as the elimination of zero-quantum coherences while retaining longitudinal two-spin order terms $2 \mathrm{I}_{z} \mathrm{~S}_{\mathrm{z}}$ for broadband excitation of long-lived (singlet) spin states [17-24], which can be used to study slow dynamics [25] and slow diffusion [26,27].

\section{Coherence encoding in diffusion experiments}

For the history of Diffusion Ordered Spectroscopy (DOSY) and earlier measurements of diffusion coefficients by magnetic resonance, the reader is referred to reviews by C.S. Johnson [28] and W.S. Price $[29,30]$. Fig. 1 shows a recent variant of the stimulated echo designed for slowly diffusing systems [13]. Typically, for complexes of ${ }^{15} \mathrm{~N}$-enriched outer-membrane proteins with detergents having molecular masses of about $45 \mathrm{kDa}, T_{1}\left({ }^{15} \mathrm{~N}\right) \approx 1 \mathrm{~s}$, whereas $T_{1}\left({ }^{1} \mathrm{H}\right) \approx 100 \mathrm{~ms}$. This makes it attractive to store the information in the form of longitudinal nitrogen magnetization. A similar advantage can be obtained with other heteronuclei. We have therefore referred to these methods as "X-STE", for "heteronuclear stimulated echoes".
Fig. 1 provides details about gradients $G_{x}$ and $G_{y}$ used to 'purge' unwanted transverse magnetization components and to select desirable echoes. The gradient $G_{1}$ purges ${ }^{15} \mathrm{~N}$ magnetization and $G_{2}$ eliminates unwanted magnetization while preserving longitudinal two-spin order $\left(2 \mathrm{H}_{z} \mathrm{~N}_{z}\right)$. The two equal $G_{3}$ gradients, like the two equal $G_{5}$ gradients later on, ensure proper refocusing under the simultaneous $180^{\circ}$ pulses applied to both ${ }^{1} \mathrm{H}$ and ${ }^{15} \mathrm{~N}$ nuclei. The $G_{4}$ gradient eliminates unwanted transverse ${ }^{1} \mathrm{H}$ and ${ }^{15} \mathrm{~N}$ magnetization components. The two equal $G_{6}$ gradients help to suppress the solvent signal by attenuating non-refocused ${ }^{1} \mathrm{H}$ magnetization in the manner of WATERGATE [4]. On the other hand, the bipolar pulse-pair (BPP) field gradients $G_{\text {encode }}$ and $G_{\text {decode }}$ in Fig. 1 allow one to encode the initial positions $\mathrm{z}(0)$ of the molecules in the first two $\tau$ intervals and to decode their final position $\mathrm{z}(\Delta)$ in the last two $\tau$ intervals, after diffusion in the long $\Delta$ period. To avoid undesirable refocusing, it is advisable (but not essential, as discussed below) to apply the $G_{z}$ field gradients in a direction that is orthogonal to the other gradients $G_{1}-G_{6}$. A clear advantage of triple axis gradient probes is that one can apply simultaneously the variable bipolar gradients $G_{\text {decode }}$ and the constant WATERGATE gradients $G_{6}$. With single-axis gradient probes where all gradients must be applied along the same direction, simultaneous gradients would be difficult to implement for several reasons. First, it is necessary to generate intense encoding and decoding gradients in order to limit the duration of the diffusion delay and to measure small diffusion coefficients. The strongest encoding and decoding gradients $G_{z}$ are often close to the maximum allowed amplitudes. Intense WATERGATE gradients $G_{6}$ are also required to ensure proper suppression of the water signal. The superposition of WATERGATE gradients with decoding gradients obviously limits their maximum intensity. As a result, a longer diffusion delay is required and the sensitivity of the experiment is reduced, while small diffusion coefficients may not be accessible. It is very likely that, at least for some values of the decoding gradients, we have $G_{6}-G_{\text {decode }} \sim 0$ and, in the absence of any gradient at that time, radiation damping during the INEPT back-transfer may decrease the efficiency of the
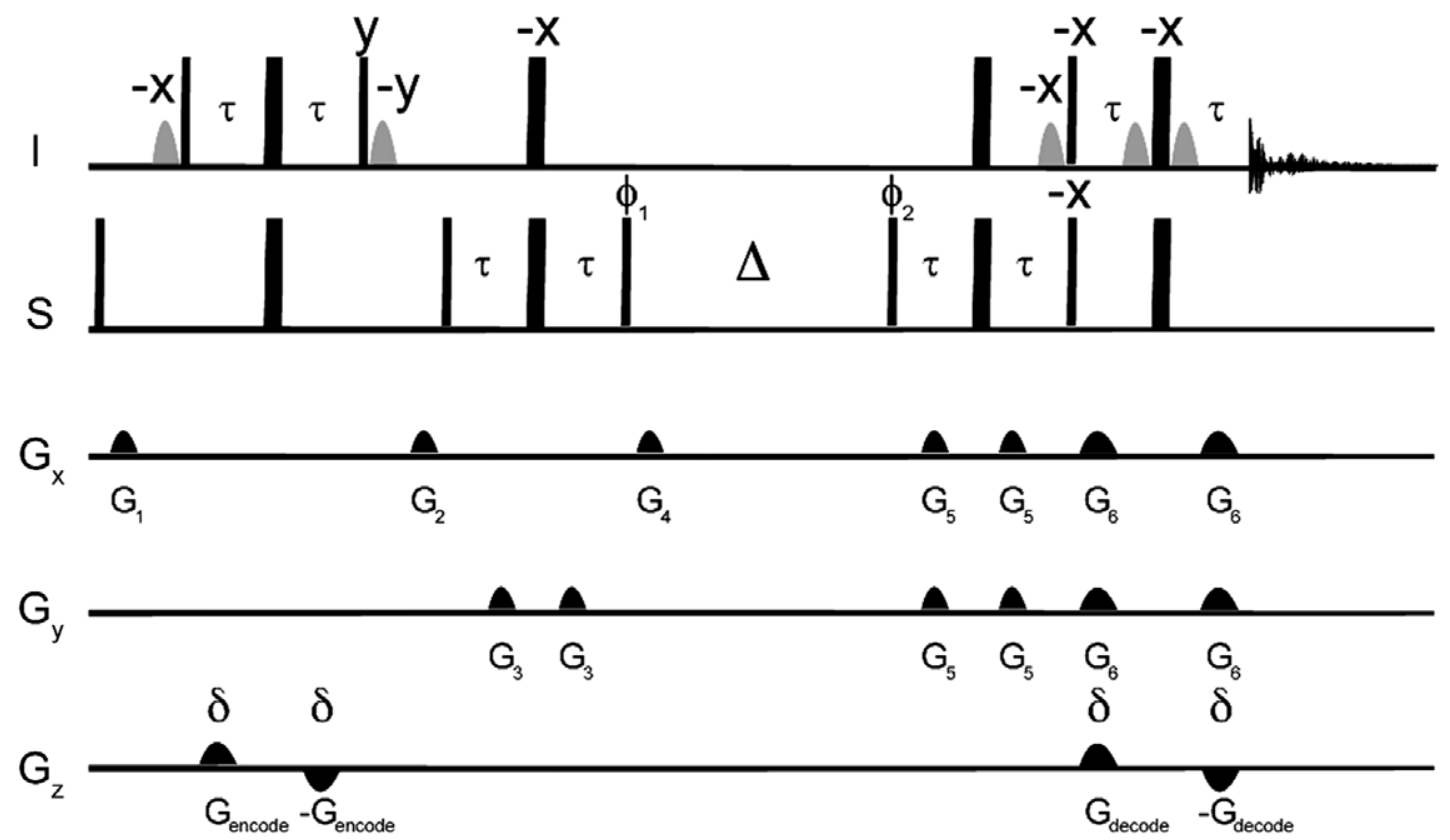

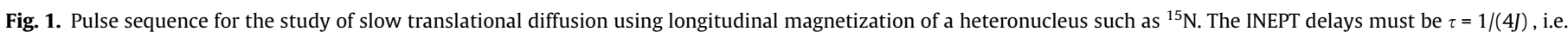

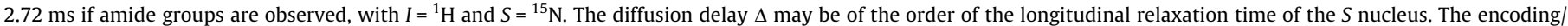

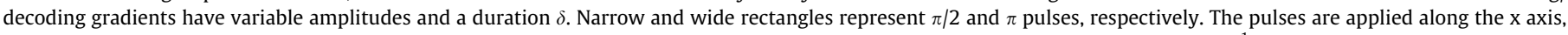

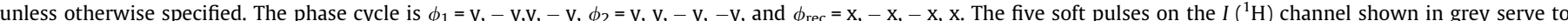

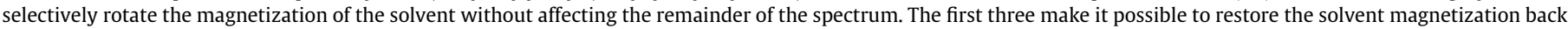
to its equilibrium position; the last two contribute to suppress the solvent magnetization in the manner of WATERGATE [4] 
suppression of the water signal. In our hands, the water signal is dramatically enhanced if the last gradient is weak. Therefore, to implement the X-STE sequence on single-axis gradient probes, the WATERGATE sequence has to be shifted after the decoding gradients (see Fig. 2). However, this leads to a loss of signal because of transverse proton relaxation. In practice, the residual water signal tends to be larger with this scheme than with triple-axis gradients. Simultaneous WATERGATE and decoding gradients along three dimensions can be designed so that the vectorial sum of the gradients may be tilted close to the magic angle, leading to an improved, more reproducible, suppression of the water signal [31].

The signal amplitudes $S$ recorded with $G_{\text {encode }}=G_{\text {decode }} \neq 0$, normalized by the signal $S_{0}$ obtained with very weak gradients $G_{\text {encode }}=G_{\text {decode }} \approx 0$, are attenuated in the following manner [13]:

$S / S_{0}=\exp \left\{-D 4 \kappa^{2}(\Delta+6 \tau)\right\}$

where $\Delta$ and $\tau$ are delays in Fig. $1, \kappa=\gamma \mathrm{s} G_{\text {encode }} \delta$, the factor $4=2^{2}$ expresses the fact that each bipolar pulse pair comprises two gradient pulses, $\gamma$ is the ${ }^{1} \mathrm{H}$ gyromagnetic ratio, $0<s<1$ is a factor that describes the shape of the gradient pulses, $\delta$ is their duration, and $D$ is the diffusion coefficient, which can be determined from the Gaussian curves $S / S_{0}$ observed when varying the maximum gradient amplitude $G_{\text {encode }}^{\max }=G_{\text {decode }}^{\max }$.

At the request of users of cryoprobes equipped with a single gradient, who wanted to implement the X-STE method, we have modified the sequence of Fig. 1, so that all PFG's can be applied along the same axis (see Fig. 2). To avoid undesirable refocusing, however, it is essential to use $G_{z}$ field gradients with different amplitudes. One should fulfill the inequalities $G_{6} \neq G_{5} \neq G_{4} \neq G_{3} \neq G_{2} \neq$ $G_{1} \neq G_{\text {encode }}$ for each of the amplitudes $G_{\text {encode }}$ employed i.e., for every experimental point in the diffusion profile. Such experiments are of course more demanding to set up than those designed for triple gradients, the sensitivity is lower, the water suppression less efficient and artefacts may result from accidental refocusing effects. Such artefacts may be hard to identify if they appear at the same chemical shift and with the same phase as naturally occurring signals. Thus, for X-STE methods as well as for some more conventional DOSY sequences, the availability of triple gradients is not essential but extremely beneficial.

\section{Suppression of zero-quantum coherences}

In many NMR experiments, the excitation of specific coherences over a broad range of shifts and couplings may be necessary. In the case of homonuclear spin systems, it is difficult to discriminate between zero-quantum coherences and longitudinal two-spin terms using radio-frequency pulses alone. Pulsed field gradients (PFGs) can make this distinction, and the best option is to use three orthogonal gradients. The amplitudes and lengths of the gradients must be set with care in order to achieve the desired selection, and they depend on other experimental parameters, such as the amplitudes and durations of frequency-swept pulses. The optimisation of the gradient amplitude is performed experimentally, as described in the following. The gradient calibration methods described herein can be beneficial for a wide range of experiments, ranging from classical sequences designed for the study of diffusion, cross-relaxation and exchange effects to broadband singletstate exchange spectroscopy.

The elimination of single- and double-quantum coherences is straightforward when a gradient is applied, as their precession rates $v_{\mathrm{SQ}}=v_{\mathrm{I}}$ or $v_{\mathrm{S}}$ and $v_{\mathrm{DQ}}=v_{\mathrm{I}}+v_{\mathrm{S}}$ strongly depend on the local field $B_{z}(\mathbf{r})$, so that these coherences rapidly dephase across the sample in the presence of a gradient. On the contrary, the precession frequency of a zero-quantum coherence (ZQC), $v_{\mathrm{ZQ}}=v_{\mathrm{I}}-v_{\mathrm{S}}$, does not depend on the typical gradient strengths. So far, the most effective solution to eliminate zero-quantum coherences in two-spin systems has proven to be an ingenious method that relies on a combination of a frequency-modulated 'Chirp' pulse with a pulsed field gradient [32,33].

This building block, which we shall henceforth refer to as the Thrippleton-Keeler (TK) filter, works in the following manner: when the precession frequencies are very similar, as is the case for zero-quantum coherences, the phases are scrambled by ensuring different evolution times for various voxels in a sample exposed to a field gradient. A considerable dephasing can be obtained by applying $\pi$ refocusing pulses at different moments in time to various ZQC components precessing at different frequencies because of the field gradient. To achieve this effect, a frequency-swept $\pi$ pulse is applied in conjunction with the gradient.

The range of frequencies swept by the pulse has to match the range induced by the gradient, and to this effect the filter has to be calibrated. The standard calibration of the TK filter [33] relies on verifying that the resonances are properly inverted by a frequency-swept Chirp pulse [34] in presence of the gradient. A 1D spectrum recorded after the filter has to have opposite sign with respect to the spectrum recorded without the filter. Although his method is straightforward, it does not measure the effect of the filter on its intended target, i.e., on zero-quantum coherence. A sequence of pulses is required to convert $\mathrm{ZQ}$ coherence into detectable signals, but manipulations before and after the application of the filter might lead to artifacts. We shall demonstrate a new way of optimizing the TK filter that measures the effect of the filter directly on a zero-quantum coherence. The use of three orthogonal gradients significantly improves the outcome. Prior to explaining this calibration method in the Materials and Methods section, we outline a few experiments for which the performance of the filter is critical.

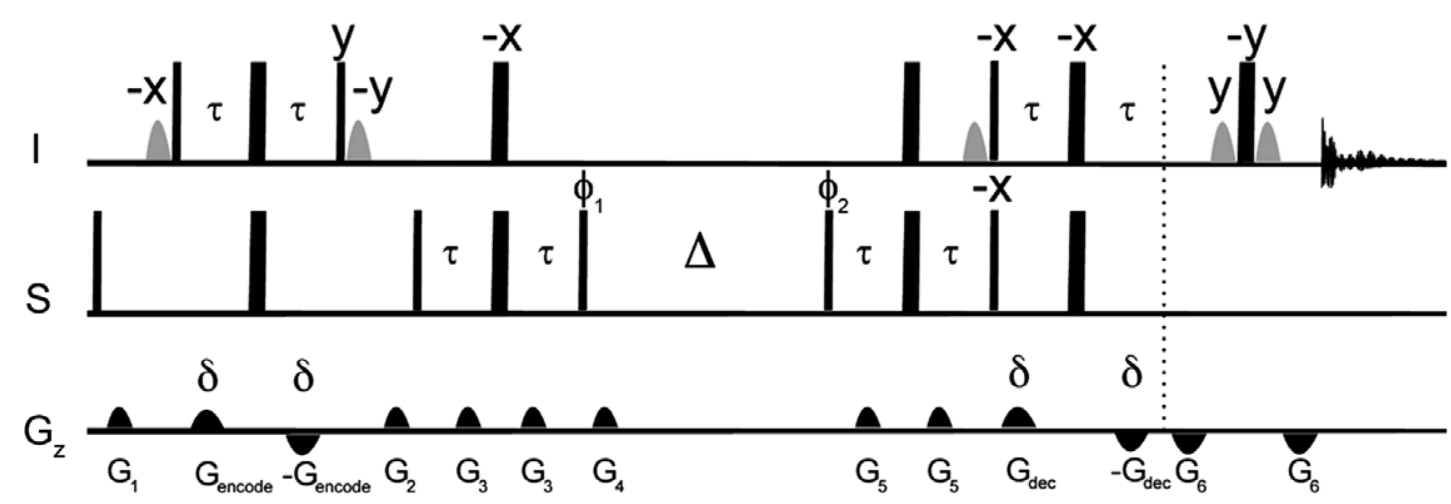

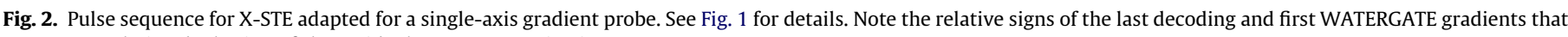
ensure cumulative dephasing of the residual water magnetization. 


\subsection{Exchange spectroscopy (EXSY) and nuclear Overhauser effect spectroscopy (NOESY)}

In these ubiquitous experiments [15,16,35], the unwanted occurrence of double- and zero-quantum coherences in the mixing interval $\tau_{m}$ may lead to misleading signals known as "J-crosspeaks" [35], i.e., dispersive anti-phase signals that may obscure genuine signals. Likewise, zz-exchange spectroscopy [36] relies on the observation of longitudinal two-spin order terms $2 \mathrm{I}_{z} \mathrm{~S}_{\mathrm{z}}$ and therefore also requires the elimination of zero-quantum coherences [33]. Because of the intensive use of these sequences in biomolecular NMR, a number of methods have been proposed to rid the spectra of ZQ coherences [37-40], but none has proven as effective as the TK filter. The calibration method proposed here and the comparison of single and triple gradients are relevant for everyday applications of the ubiquitous EXSY and NOESY methods.

\subsection{Suppression of zero-quantum coherences in broadband singlet- state exchange spectroscopy (SS-EXSY)}

The elimination of zero-quantum coherences (ZQC) is also desirable in experiments using long-lived (singlet) states $[18,19,21,23-25]$ in homonuclear spin systems with arbitrary offsets and scalar couplings. Broadband excitation of singlet states in systems containing two or more spins opens new horizons for the investigation of slow dynamic processes by NMR. Long-lived states are useful for experiments to study diffusion [26,27] and slow exchange [25], and allow one to derive molecular symmetry and other properties reflected by dipole-dipole and chemical shift anisotropy interactions [24]. Singlet states can be maintained by applying a strong continuous-wave $(\mathrm{CW})$ radio-frequency $(\mathrm{RF})$ field

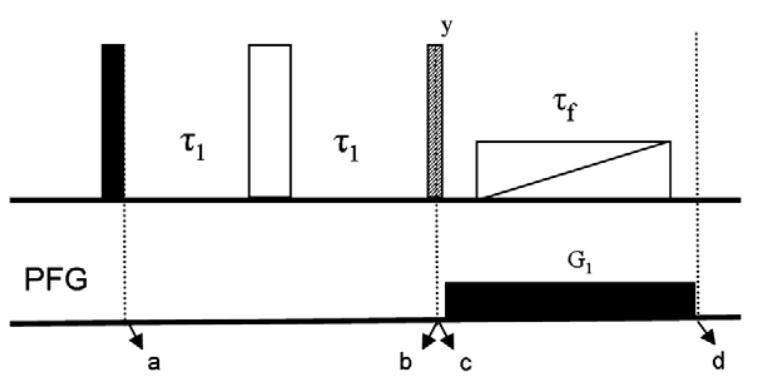

Fig. 3. Excitation of two-spin coherences $2 I_{z} S_{z}$ and $Z Q_{x}$ in homonuclear two-spin systems and elimination of $Z Q_{x}$ using a TK filter consisting of a gradient $G_{1}$ and a frequency-swept pulse of duration $\tau_{\mathrm{f}}$. The hatched, filled, and open rectangles stand for $\pi / 4, \pi / 2$, and $\pi$ pulses, respectively. The pulses are applied along the $x$ axis, unless otherwise specified.
[18,41], or by applying suitable trains of composite or shaped pulses [23]. Applying established methods to distinguish between coherence orders $[42,43]$, singlet states can be selected by using socalled isotropic filters with tetrahedral phase cycling [22]. The TK filter is the method of choice to purge coherences independently of the chemical shifts.

It has been shown [25] that singlet states may be excited over a broad range of chemical shifts and J-coupling constants by selecting longitudinal two-spin order while eliminating zero-quantum coherences at the beginning of the interval where these states are sustained by suitable RF irradiation. The filter has to be applied a second time after this interval, in order to reconvert singlet states into detectable coherences. For both selection steps, TK filters have been shown to be effective. In order to avoid accidental refocusing effects due to the combination of the two filters, it is preferable to apply the gradients along two orthogonal directions. The long lifetimes of singlet states offer straightforward means to distinguish them from other coherences. This method recommends itself whenever one is interested in the study of slow dynamic phenomena, such as exchange and diffusion. However, the singlet states can also provide information on molecular symmetry and structure [24]. For instance, if a singlet state is excited to determine the relative orientation of the chemical shift anisotropy (CSA) tensors of two nuclei, the lifetime of this state may be comparable to that of other coherences. Accurate fitting of the decay curves of the singlet state requires that other coherences be properly eliminated. In sequences used in this type of studies, where short evolution intervals are required, it is therefore essential to separate long-lived states from other coherences.

\section{Materials and methods}

\subsection{Elimination of zero-quantum coherences}

In a homonuclear pair of $J$-coupled spins $I$ and $S$, a spin-echo sequence $\left[(\pi / 2)_{x}-\tau_{1}-(\pi)_{x}-\tau_{1}\right]$ with a duration $2 \tau_{1}=1 /(2 J)$ leads to antiphase terms $2 I_{x} S_{z}+2 I_{z} S_{x}$ (at time point $b$ in Fig. 3). A $(\pi / 4)_{y}$ pulse transforms these antiphase terms into a superposition of longitudinal two-spin order, zero-quantum, and double-quantum coherences at point $c$ of the sequence in Fig. 3:

$2 I_{\mathrm{x}} S_{\mathrm{z}}+2 I_{\mathrm{z}} S_{\mathrm{x}} \rightarrow 2 I_{\mathrm{x}} S_{\mathrm{x}}-2 I_{\mathrm{z}} S_{\mathrm{z}}=Z Q_{\mathrm{x}}+D Q_{\mathrm{x}}-2 I_{\mathrm{z}} S_{\mathrm{z}}$

where the zero- and double-quantum coherences are defined as usual [44]:

$$
\begin{aligned}
& Z Q_{\mathrm{x}}=1 / 2\left(2 I_{\mathrm{x}} S_{\mathrm{x}}+2 I_{\mathrm{y}} S_{\mathrm{y}}\right) \\
& D Q_{\mathrm{x}}=1 / 2\left(2 I_{\mathrm{x}} S_{\mathrm{x}}-2 I_{\mathrm{y}} S_{\mathrm{y}}\right)
\end{aligned}
$$
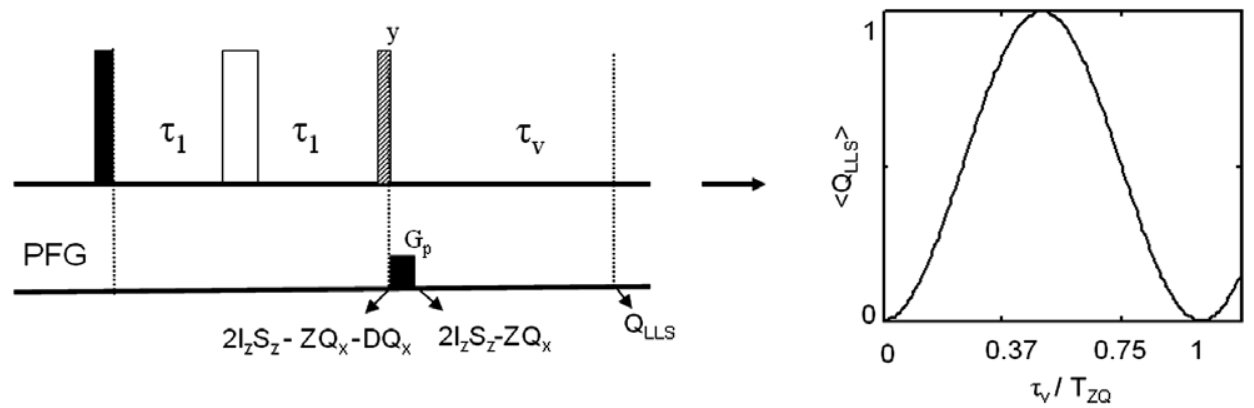

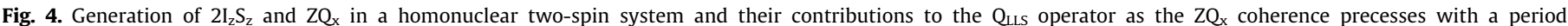

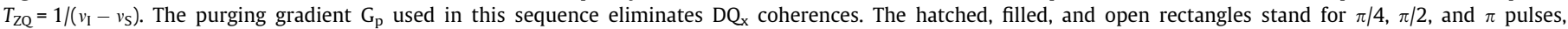
respectively. The pulses are applied along the $x$ axis, unless otherwise specified. 


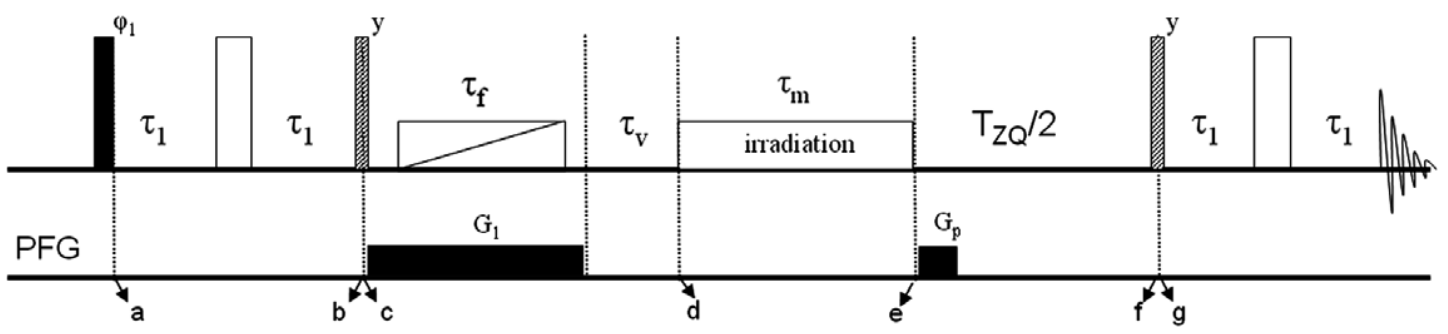

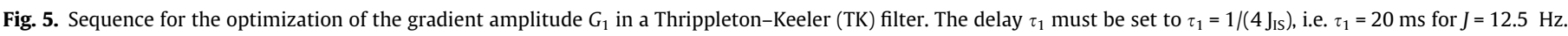

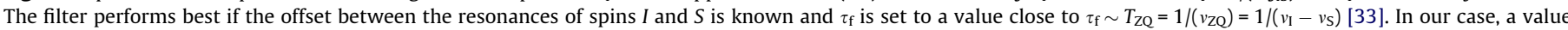

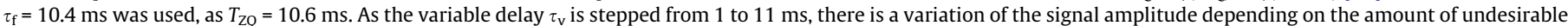

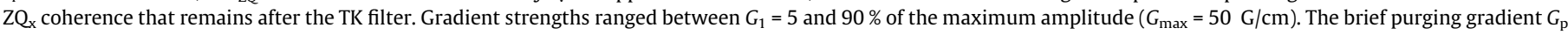

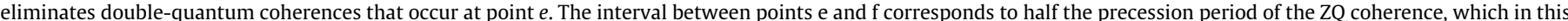

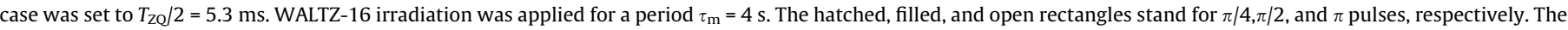
pulses are applied along the $\mathrm{x}$ axis, unless otherwise specified. The phase cycling was $\phi_{1}=\mathrm{x},-\mathrm{x}, \phi_{\mathrm{rec}}=\mathrm{x},-\mathrm{x}$.

A<smiles>[Z]CO[AsH3]</smiles>

B

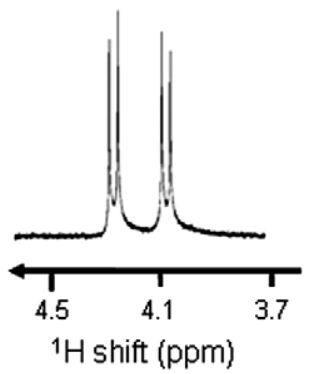

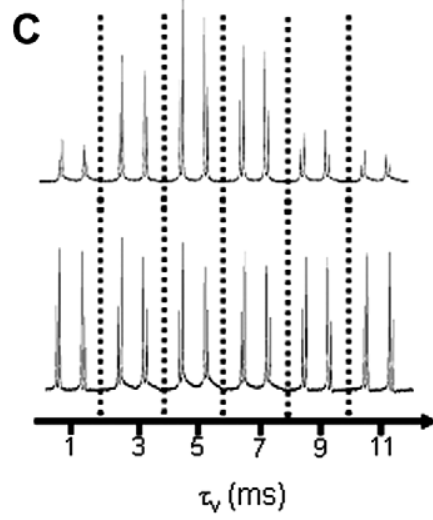

$\tau_{v}(\mathrm{~ms})$
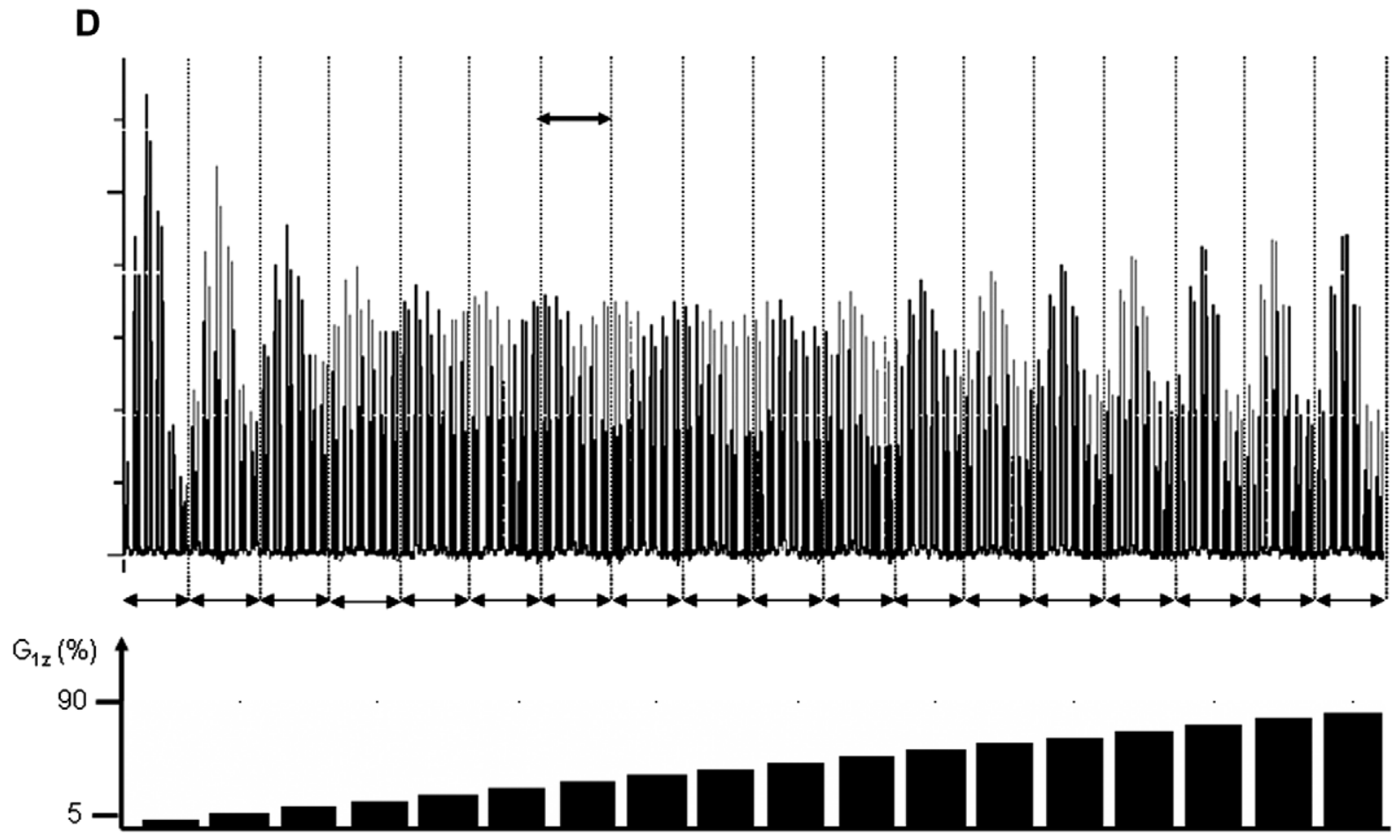

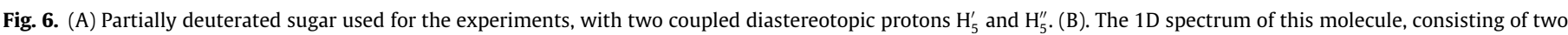

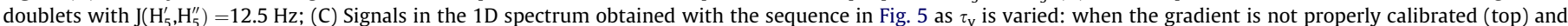

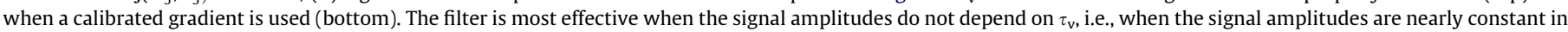

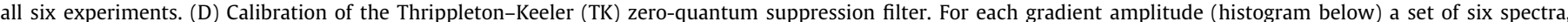

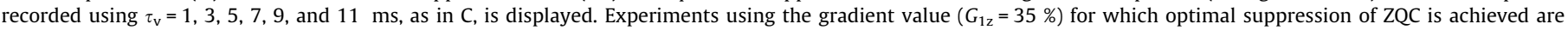
outlined by a double-headed arrow: intensities in these experiments are almost constant (independent of $\tau_{\mathrm{v}}$ ). 
In a similar manner, a mixture of $2 I_{y} S_{y}$ and $2 I_{z} S_{z}$ terms can be generated by starting with a $(\pi / 2)_{y}$ pulse, generating antiphase terms $2 I_{y} S_{z}+2 I_{z} S_{y}$ and subsequently applying a $(\pi / 4)_{x}$ pulse. These terms can be readily inter-converted, e.g., a $(\pi / 2)_{x}$ pulse inter-converts $2 I_{y} S_{y}$ and $2 \mathrm{I}_{z} S_{z}$.

In the sequence in Fig. 3, the TK filter in the interval between points $c$ and $d$ consists of a combination of a frequency-swept pulse and a gradient $\mathrm{G}_{1}$. Its effect [33] is to eliminate all in-plane terms, leaving populations $I_{z}$ and $S_{z}$ and longitudinal two-spin order $2 I_{z} S_{z}$ unaffected. Therefore, at point $d$ in Fig. 3 the only remaining term is $2 I_{z} S_{z}$.

Long-lived states LLS (or, equivalently, singlet states, in systems that contain only two spins) can be described by combinations of operator products:

$Q_{\text {LLS }}=-\frac{2}{\sqrt{3}} \vec{I} \cdot \vec{S}=-\frac{2}{\sqrt{3}}\left(I_{\mathrm{x}} S_{\mathrm{x}}+I_{\mathrm{y}} S_{\mathrm{y}}+I_{\mathrm{z}} S_{\mathrm{z}}\right)=-\frac{2}{\sqrt{3}}\left(I_{\mathrm{z}} S_{\mathrm{z}}+Z Q_{\mathrm{x}}\right)$

The last expression shows the importance of the relative signs of the longitudinal two-spin order $2 I_{z} S_{z}$ and the zero-quantum $Z Q_{x}$ terms. Ideally, both terms should have equal projections [45] on $Q_{L L S}$ :

$P=\operatorname{Tr}\left(2 I_{\mathrm{z}} S_{\mathrm{z}}^{\dagger} \cdot Q_{\mathrm{LLS}}\right)=\operatorname{Tr}\left(Z Q_{\mathrm{x}}^{\dagger} \cdot Q_{\mathrm{LLS}}\right)=-\frac{1}{\sqrt{3}}$
After an excitation step similar to the one outlined in Fig. 3 (time points a to $c$ ), the $Z Q_{x}$ term has a sign opposite to the $2 I_{z} S_{z}$ term (see Fig. 4). Subsequently, the $Z Q_{x}$ term may acquire an arbitrary phase during free precession, so that its contribution to the singlet state LLS may be additive or subtractive (Fig. 4).

This property can be used in order to calibrate the TK filter in Fig. 3 by inserting a variable delay $\tau_{v}$ after the filter before switching on the RF irradiation that generates and maintains the singlet state. If the signal amplitude at the outcome of such a sequence depends on $\tau_{v}$, this indicates incomplete suppression of the $Z Q_{x}$ coherence by the TK filter. On the other hand, a signal of constant amplitude regardless of $\tau_{\mathrm{v}}$ indicates that the filter is correctly calibrated, so that the $Z Q_{x}$ is properly suppressed, and the singlet state originates exclusively from $2 I_{z} S_{z}$ terms.

\section{Experimental}

All experiments were carried out on $500 \mathrm{MHz}\left(B_{0}=11.75 \mathrm{~T}\right)$ spectrometers in Fällanden and Lausanne, both equipped with prototypes of cryoprobes with three orthogonal gradients. The test sample was a monosaccharide with a five-membered furanose ring where all hydrogen atoms - except those in positions
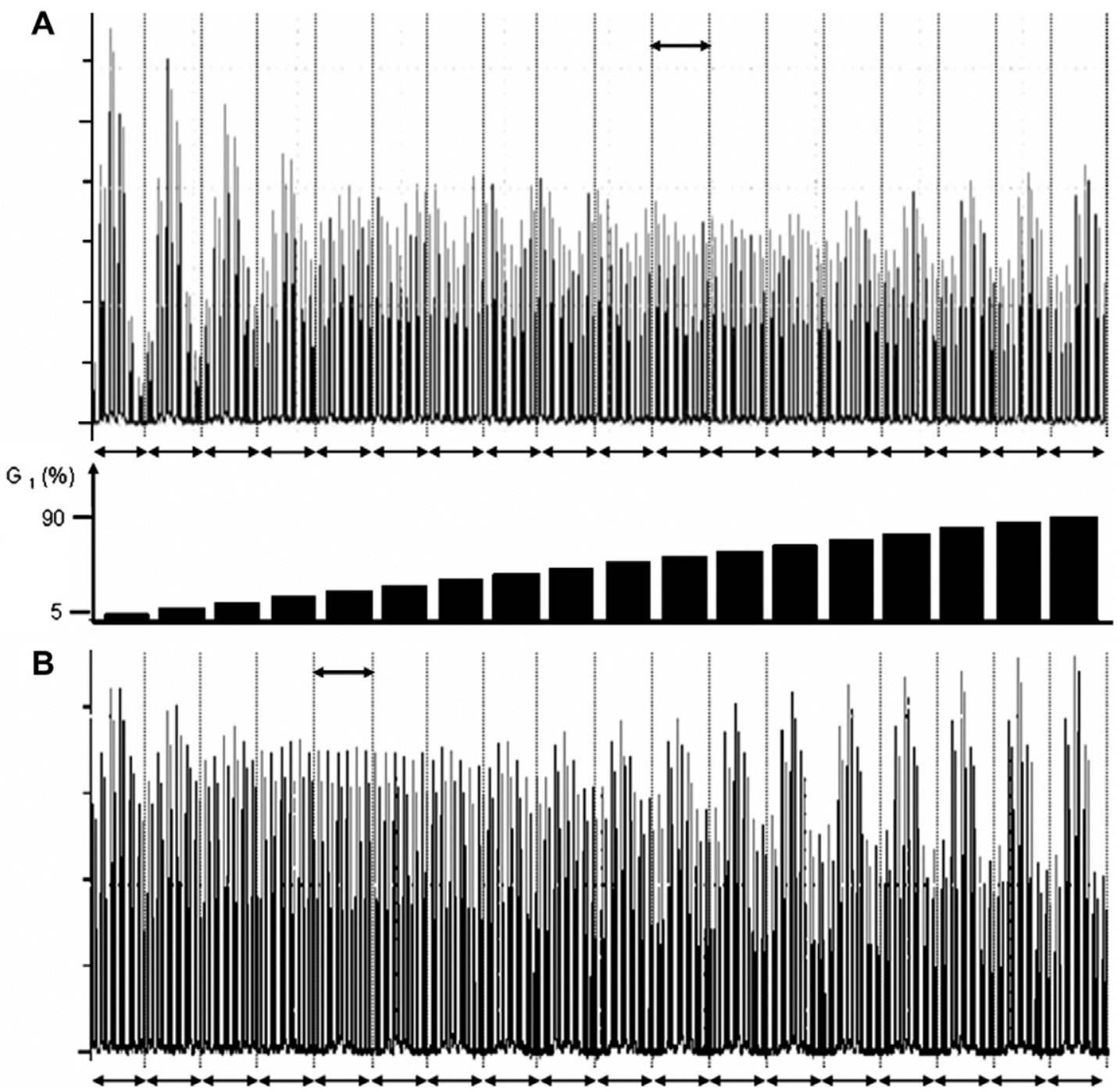

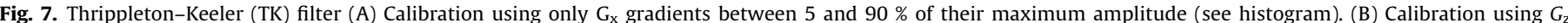

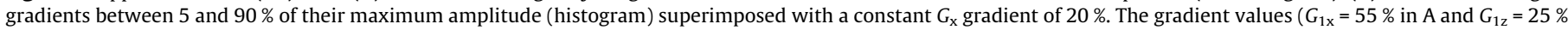
in $\mathrm{B}$, respectively) for which optimal suppression of ZQC and, therefore, almost constant profiles are obtained, are evidenced by double-headed arrows. 
$\mathrm{H}_{5}^{\prime}$ and $\mathrm{H}_{5}^{\prime \prime}$ - were replaced by deuterium atoms so that the lifetime of the singlet state was $T_{\mathrm{S}} \sim 26 \mathrm{~s}$, while the longitudinal relaxation time, $T_{1}$, was $0.7 \mathrm{~s}$, as described in more detail elsewhere [25]. The test sample was contained in a Shigemi tube with an useful height of $12 \mathrm{~mm}$ and an outer diameter of $5 \mathrm{~mm}$. The concentration of the sample, dissolved in deuterated DMSO, was $\sim 10 \mathrm{mM}$ and its temperature $300 \mathrm{~K}$. Two transients were acquired with an acquisition time of $0.8 \mathrm{~s}$, a spectral width of $10.3 \mathrm{kHz}(20.6 \mathrm{ppm})$ and a relaxation delay of $30 \mathrm{~s}$, preceded by two 'dummy scans', without acquiring any signal. In the TK filters, 'Chirp' pulses with linear frequency sweeps were used, with a maximum amplitude $v_{1}^{\max }=1.82 \mathrm{kHz}$, apodised by a sine function during the first and last $10 \%$ of their duration, and a frequency sweep range of $40 \mathrm{kHz}[33,46]$. The maximum allowed gradient amplitude is the same in all three directions of space: $G_{x, \max }=G_{\mathrm{y}, \max }=$ $G_{z, \max }=50 \mathrm{G} / \mathrm{cm}$, inducing a frequency spread for protons in the sample of $212 \mathrm{kHz} / \mathrm{cm}$. A WALTZ-16 [47] sequence, with a constant RF amplitude $v_{1}=3.13 \mathrm{kHz}$ was used to maintain the singlet states during an interval $\tau_{\mathrm{m}}=4 \mathrm{~s}$. The total duration of the filter optimisation process, using six experiments for each gradient amplitude and 18 increments of the gradient amplitude between $5 \%$ and $90 \%$ of $G_{\max }$ was $4 \mathrm{~h}$.

\section{Results and discussion}

An imperfect cancellation of $Z_{\mathrm{x}}$ terms after point $c$ of the sequence in Fig. 3 results in variations of the singlet state populations at the beginning of the $\mathrm{RF}$ irradiation interval. This is due to destructive interference of the longitudinal two-spin order and zero-quantum coherences at point $d$.

We have calibrated the filter described in Materials and Methods using a sequence that excites both $2 I_{z} S_{z}$ and $Z Q_{x}$. The latter is partly eliminated by the filter, while the former is transformed into a long-lived (singlet) state and subsequently re-converted into detectable magnetization (Fig. 5).

As the delay $\tau_{\mathrm{v}}$ is varied, depending on the amount of $Z Q_{\mathrm{x}}$ coherence that survives after the TK filter, there is a variation of the signal amplitude. The sequence was used with $G_{1}$ gradients between 5 and $90 \%$ of their maximum allowed amplitude, recording for each gradient amplitude 6 spectra, using $\tau_{\mathrm{v}}=1,3$, $5,7,9$, and $11 \mathrm{~ms}$, as described in the caption to Fig. 6 . Note that the property outlined in Fig. 4 is used to achieve a constructive interference between $2 I_{z} S_{z}$ and $Z Q_{x}$ between points eand $f$ of the sequence, by setting this interval to half of the precession period of the zero-quantum coherence, $T_{\mathrm{ZO}} / 2=1 / 2\left[1 /\left(v_{\mathrm{I}}-v_{\mathrm{S}}\right)\right]$; for instance, in our case, a frequency difference $v_{\mathrm{I}}-v_{\mathrm{S}}=94 \mathrm{~Hz}$ led us

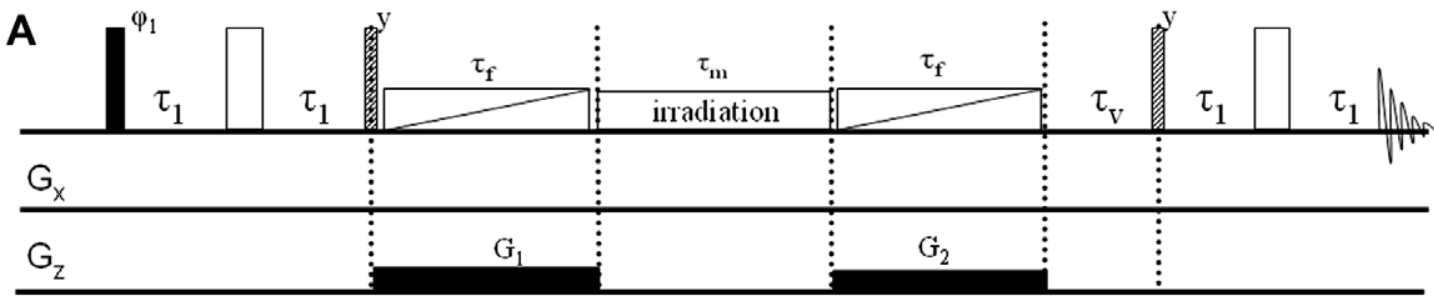

B
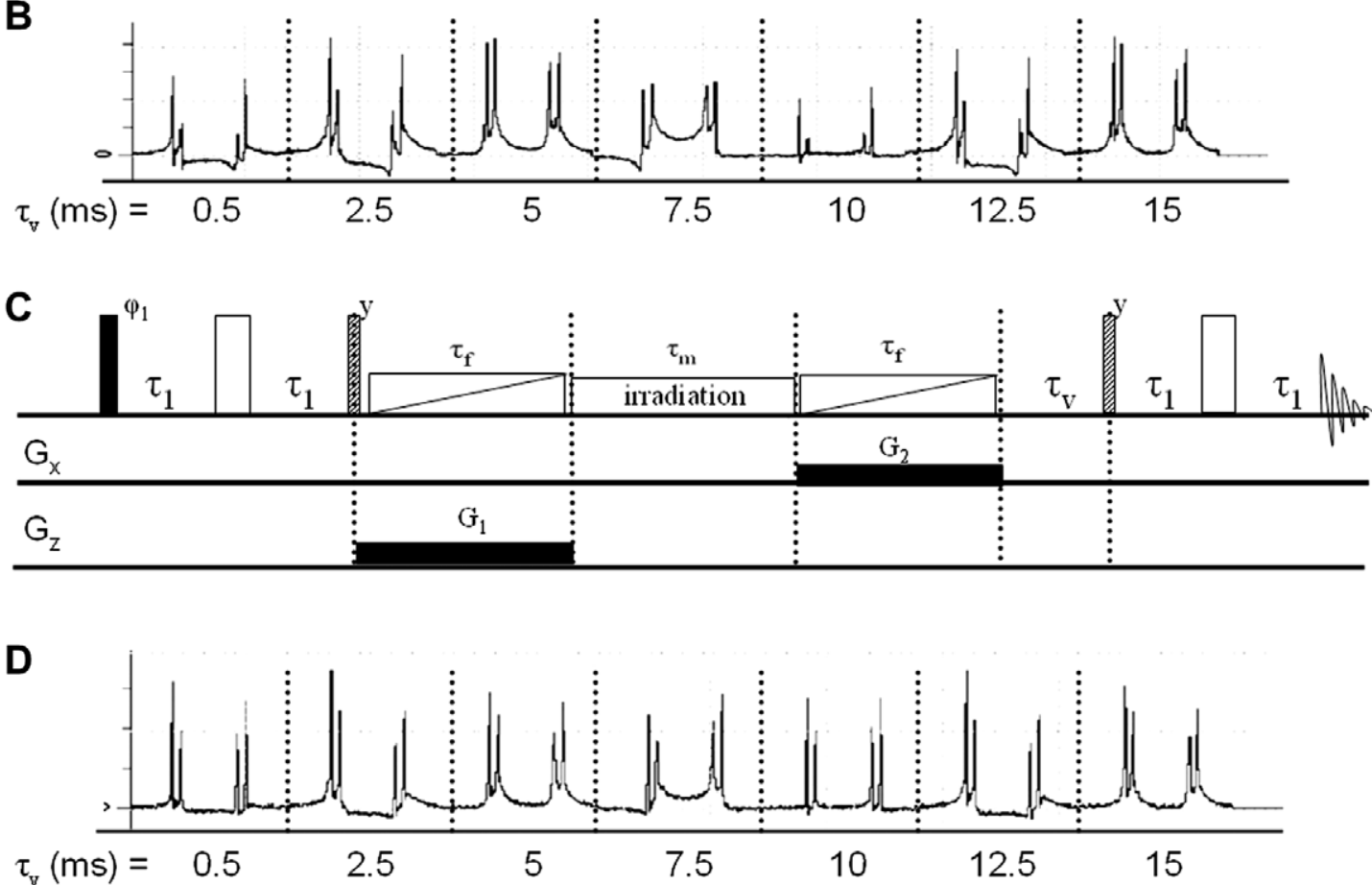

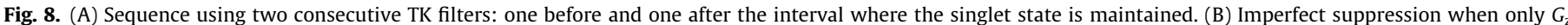

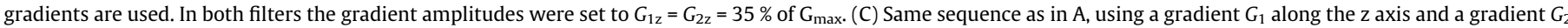

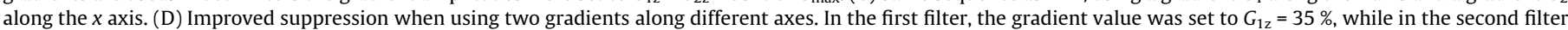

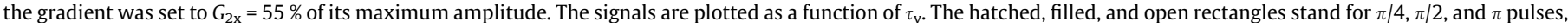
respectively. The pulses are applied along the $\mathrm{x}$ axis, unless otherwise specified. The phase cycling was $\phi_{1}=\mathrm{x},-\mathrm{x}, \phi_{\text {rec }}=\mathrm{x},-\mathrm{x}$. 
to set $T_{z O} / 2=5.3 \mathrm{~ms}$. The results of this calibration protocol are shown in Fig. 6.

The smallest $\tau_{\mathrm{v}}$-dependent variations of the signal amplitudes occur when the gradient $G_{1}$ has an amplitude corresponding to $35 \%$ of its maximum $\left(G_{\max }=50 \mathrm{G} / \mathrm{cm}\right)$. This gradient amplitude therefore corresponds to an optimal calibration of the TK filter. It is worth noting that the filter needs to be calibrated only once for a given probe. The total duration of the $6 \times 18=108$ experiments needed for calibration was $4 \mathrm{~h}$, owing to the high sensitivity of the cryoprobe (2 transients/experiment were sufficient).

We have also explored the performance of the TK filter when the gradient is applied along a different axis. Using the sequence in Fig. 5 with $G_{\mathrm{x}}$ rather than $G_{\mathrm{z}}$ gradients, it can be expected that different gradient amplitudes will be needed to optimise the TK filter. As the same 'Chirp' pulse was used with both $G_{\mathrm{x}}$ and $G_{\mathrm{z}}$ gradiens, a stronger gradient value was required to create the same distribution of frequencies across the width ( $x$ dimension) of the sample tube, which is smaller than its height ( $z$ dimension). The characteristics of the tube (e.g., whether a Shigemi tube is used) obviously make a difference. It was found (Fig. 7) that the efficiency of the filter was maximal when the $G_{x}$ gradient had an amplitude of $55 \%$ of $G_{\text {max }}$. It should be noted that the difference between the optimal values $G_{1 z}=35 \%$ (Fig. 6D) and $G_{1 x}=55 \%$ of $G_{\max }$ (Fig. 7A) is mainly due the difference between the length and the diameter of the sample. When a superposition of $G_{x}$ and $G_{z}$ gradients was evaluated while maintaining the $G_{x}$ gradient constant (20\% of $G_{\max }$ ), the TK filter was found to perform best for an amplitude $G_{z}$ of $25 \%$. This combination of two orthogonal gradients leads to signal intensities that are nearly independent of $\tau_{v}$, i.e., a better performance than can be attained when only a $G_{z}$ gradient is used. The vector sum of amplitudes of the $G_{\mathrm{x}}$ and $G_{\mathrm{z}}$ gradients that optimises the performance of the filter is a little smaller than the amplitude of the $\mathrm{z}$ gradient alone that yields the same effect.

We have shown [23] that, using adequate irradiation methods with shaped pulses with RF peak amplitudes of up to $16 \mathrm{kHz}$, the frequency bandwidth over which singlet states can be sustained can be extended to about $\pm 12 \mathrm{kHz}$. The excitation of singlet states over a bandwidth of this order of magnitude (i.e., independently of the chemical shifts of the spins $I$ and $S$ ) implies that the $Z Q$ must be eliminated by a proper calibration of the TK filters over the same bandwidth. The elimination of the $Z Q_{x}$ coherences has to be performed twice, first prior to the generation of the singlet states, and again when they are converted back into observable coherences. Indeed, a sum of $Z Q_{x}$ and $\mathrm{I}_{z} S_{z}$ coherences resulting from the $Q_{L L S}$ operator after the irradiation period gives no observable antiphase signals if a $(\pi / 4)_{\mathrm{y}}$ is applied immediately, but only if a suitable interval, $\tau_{\mathrm{v}}$, or a TK filter are inserted. We have tested whether the application of two TK filters (in the singlet-state excitation and back-conversion steps) using two $G_{z}$ gradients (i.e., along the same axis) results in the generation of artefacts. Even when both gradients are set to their optimum values, some artefacts survive after the second filter (Fig. 8) in this situation.

The application of filters using two orthogonal gradients $G_{z}$ and $G_{\mathrm{X}}$ resulted in a clean selection of signals (Figs. 7 and $8 \mathrm{C}$ and D) that were largely independent of the delay $\tau_{\mathrm{v}}$ inserted prior to the last $\pi / 4$ pulse, indicative of the fact that little $Z Q_{x}$ coherences survived.

\section{Conclusions}

It has been shown that probes with triple gradients are of great advantage for the accurate determination of slow diffusion constants with the X-STE method. A reliable calibration of Thrippleton-Keeler (TK) filters, designed to eliminate zero-quantum coherences, has been presented. By calibrating these filters against their intended target, i.e., against zero-quantum coherences, it has been shown that their performance is improved when at least two orthogonal gradients are available. These methods will be useful to optimize the zero-quantum elimination in EXSY and NOESY sequences, in z-COSY, in zz-exchange spectroscopy, in singlet-state DOSY and singlet-state exchange spectroscopy. It can be concluded that probes endowed with triple gradients should be preferred over probes that are equipped only with longitudinal gradients.

\section{Acknowledgments}

This work was supported by the Swiss Commission for Technology and Innovation (CTI), the Swiss National Science Foundation (FNRS), the Ecole Polytechnique Fédérale de Lausanne (EPFL), and the French CNRS.

\section{References}

[1] P.T. Callaghan, Principles of nuclear magnetic resonance microscopy, Oxford University Press, 1991.

[2] E.O. Stejskal, J.E. Tanner, Spin diffusion measurements-spin echoes in presence of a time-dependent field gradient, J. Chem. Phys. 42 (1965) 288-292.

[3] J.E. Tanner, Use of stimulated echo in NMR-diffusion studies, J. Chem. Phys. 52 (1970) 2523-2526.

[4] M. Piotto, V. Saudek, V. Sklenar, Gradient-tailored excitation for singlequantum NMR-spectroscopy of aqueous solutions, J. Biomol. NMR 2 (1992) 661-665.

[5] A.A. Maudsley, A. Wokaun, R.R. Ernst, Coherence transfer echoes, Chem. Phys. Lett. 55 (1978) 9-14.

[6] D.R. Muhandiram, T. Yamazaki, B.D. Sykes, L.E. Kay, Measurement of H-2 $T_{1}$ and $T_{1 \text { rho }}$ relaxation-times in uniformly C-13-labeled and fractionally $\mathrm{H}-2$-labeled proteins in solution, J. Am. Chem. Soc. 117 (1995) 11536-11544.

[7] L. Frydman, T. Scherf, A. Lupulescu, The acquisition of multidimensional NMR spectra within a single scan, Proc. Natl. Acad. Sci. 99 (2002) 15858-15862.

[8] P. Pelupessy, Adiabatic single scan two-dimensional NMR spectroscopy, J. Am. Chem. Soc. 125 (2003) 12345-12350.

[9] W. Richter, S.H. Lee, W.S. Warren, Q.H. He, Imaging with intermolecular multiple-quantum coherences in solution nuclear magnetic resonance, Science 267 (1995) 654-657.

[10] M.H. Levitt, Demagnetizing field effects in two-dimensional solution NMR, Concepts Magn. Reson. 8 (1996) 77-103.

[11] S. Ahn, W.S. Warren, S. Lee, Quantum treatment of intermolecular multiplequantum coherences with intramolecular J coupling in solution NMR, J. Magn. Reson. 128 (1997) 114-129.

[12] D.G. Cory, W.S. Veeman, Magnetic-field gradient imperfections in NMR imaging of rotating solids, J. Magn. Reson. 82 (1989) 374-381.

[13] F. Ferrage, M. Zoonens, D.E. Warschawski, J.L. Popot, G. Bodenhausen, Slow diffusion of macromolecular assemblies by a new pulsed field gradient NMR method, J. Am. Chem. Soc. 125 (2003) 2541-2545; corrigendum to this article, J. Am. Chem. Soc. 126 (2004) 5654

[14] B.H. Meier, R.R. Ernst, Elucidation of chemical-exchange networks by 2dimensional NMR spectroscopy-heptamethylbenzenonium ion, J. Am. Chem. Soc. 101 (1979) 6441-6442.

[15] J. Jeener, B.H. Meier, P. Bachmann, R.R. Ernst, Investigation of exchange processes by 2-dimensional NMR spectroscopy, J. Chem. Phys. 71 (1979) 4546-4553.

[16] A. Kumar, R.R. Ernst, K. Wüthrich, A two-dimensional nuclear overhauser enhancement (2D NOE) experiment for the elucidation of complete protonproton cross-relaxation networks in biological macromolecules, Biochem. Biophys. Res. Commun. 95 (1980) 1-6.

[17] M. Carravetta, O.G. Johannessen, M.H. Levitt, Beyond the $T_{1}$ limit: singlet nuclear spin states in low magnetic fields, Phys. Rev. Lett. 92 (2004) 153003153004.

[18] M. Carravetta, M.H. Levitt, Long-lived nuclear spin states in high-field solution NMR, J. Am. Chem. Soc. 126 (2004) 6228-6229.

[19] M. Carravetta, M.H. Levitt, Theory of long-lived nuclear spin states in solution nuclear magnetic resonance. I. Singlet states in low magnetic field, J. Chem. Phys. 122 (2005) 214505-214506.

[20] G. Pileio, M. Concistrè, M. Carravetta, M.H. Levitt, Long-lived nuclear spin states in the solution NMR of four-spin systems, J. Magn. Reson. 182 (2006) 353-357.

[21] G. Pileio, M.H. Levitt, J-Stabilisation of singlet states in the solution NMR of multiple-spin systems, J. Magn. Reson. 187 (2007) 141-145.

[22] G. Pileio, M.H. Levitt, Isotropic filtering using polyhedral phase cycles. Application to singlet-state NMR, J. Magn. Reson. 191 (2008) 148-155.

[23] R. Sarkar, P. Ahuja, D. Moskau, P.R. Vasos, G. Bodenhausen, Extending the scope of singlet-state spectroscopy, Chem. Phys. Chem. 8 (2007) 26522656.

[24] P. Ahuja, R. Sarkar, P.R. Vasos, G. Bodenhausen, Molecular properties determined from the relaxation of long-lived spin states, J. Chem. Phys. 127 (2007) 134112 
[25] R. Sarkar, P.R. Vasos, G. Bodenhausen, Singlet-state exchange NMR spectroscopy for the study of very slow dynamic processes, J. Am. Chem. Soc. 129 (2007) 328-334

[26] S. Cavadini, J. Dittmer, S. Antonijevic, G. Bodenhausen, Slow diffusion by singlet-state NMR spectroscopy, J. Am. Chem. Soc. 127 (2005) 15744-15748.

[27] S. Cavadini, P.R. Vasos, Singlet states open the way to longer time-scales in the measurement of diffusion by NMR spectroscopy, Concepts Magn. Reson. 32A (2008) 68-78.

[28] C. Johnson, Diffusion ordered nuclear magnetic resonance spectroscopy: principles and applications, Progr. Nucl. Magn. Reson. Spectrosc. 34 (1998) 203-256.

[29] W.S. Price, Pulsed-field gradient nuclear magnetic resonance as a tool for studying translational diffusion. 1. Basic theory, Concepts Magn. Reson. 9 (1997) 299-336.

[30] W.S. Price, Pulsed-field gradient nuclear magnetic resonance as a tool for studying translational diffusion: part II. Experimental aspects, Concepts Magn. Reson. 10 (1998) 197-237.

[31] Y.Y. Lin, N. Lisitza, S.D. Ahn, W.S. Warren, Resurrection of crushed magnetization and chaotic dynamics in solution NMR spectroscopy, Science 290 (2000) 118-121.

[32] M.J. Thrippleton, R.A.E. Edden, J. Keeler, Suppression of strong coupling artefacts in J-spectra, J. Magn. Reson. 174 (2005) 97-109.

[33] M.J. Thrippleton, J. Keeler, Elimination of zero-quantum interference in twodimensional NMR spectra, Angew. Chem. Int. Ed. 42 (2003) 3938-3941.

[34] J.M. Böhlen, G. Bodenhausen, Experimental aspects of chirp NMRspectroscopy, J. Magn. Reson. Ser. A 102 (1993) 293-301.

[35] S. Macura, Y. Huang, D. Suter, R.R. Ernst, Two-dimensional chemical-exchange and cross-relaxation spectroscopy of coupled nuclear spins, J. Magn. Reson. 43 (1981) 259-281.
[36] G. Wagner, G. Bodenhausen, N. Müller, M. Rance, O.W. Sørensen, R.R. Ernst, K. Wüthrich, Exchange of 2-spin order in nuclear magnetic-resonanceseparation of exchange and cross-relaxation processes, J. Am. Chem. Soc. 107 (1985) 6440-6446.

[37] S. Macura, R.R. Ernst, The study of solvent-solute interactions by twodimensional NMR spectroscopy, Period. Biol. 83 (1981) 87-93.

[38] S. Macura, K. Wüthrich, R.R. Ernst, Separation and suppression of coherent transfer effects in two-dimensional NOE and chemical-exchange spectroscopy, J. Magn. Reson. 46 (1982) 269-282.

[39] M. Rance, G. Bodenhausen, G. Wagner, K. Wüthrich, R.R. Ernst, A systematicapproach to the suppression of J-cross peaks in 2D exchange and 2D NOE spectroscopy, J. Magn. Reson. 62 (1985) 497-510.

[40] M. Baur, H. Kessler, Novel suppression of the diagonal signals in the NOESY experiment, Magn. Res. Chem. 35 (1997) 877-882.

[41] K. Gopalakrishnan, G. Bodenhausen, Lifetimes of singlet-states under coherent off-resonance irradiation in NMR spectroscopy, J. Magn. Reson. 182 (2006) 254-259.

[42] G. Bodenhausen, H. Kogler, R.R. Ernst, Selection of coherence-transfer pathways in NMR pulse experiments, J. Magn. Reson. 58 (1984) 370-388.

[43] M.H. Levitt, P.K. Madhu, C.E. Hughes, Cogwheel phase cycling, J. Magn. Reson. 155 (2002) 300-306.

[44] P. Hore, J. Jones, S. Wimperis, NMR, the Toolkit, Oxford University Press, Oxford, 2000.

[45] R.R. Ernst, G. Bodenhausen, A. Wokaun, Principles of nuclear magnetic resonance in one and two dimensions, Oxford University Press, Oxford, 1987

[46] R. Fu, G. Bodenhausen, Broad-band decoupling in NMR with frequencymodulated chirp pulses, Chem. Phys. Lett. 245 (1995) 415-420.

[47] A.J. Shaka, J. Keeler, T. Frenkiel, R. Freeman, An improved sequence for broadband decoupling-WALTZ-16, J. Magn. Reson. 52 (1983) 335-338. 